\title{
The overlap between low self-esteem and anxiety/depression in CAMHS
}

\author{
By Loades, M.E. ${ }^{1,2}$
}

\section{Affiliations:}

1 Department of Psychology, University of Bath

2 Bristol Medical School, University of Bristol

\section{Contact details:}

Maria Loades,

Department of Psychology,

University of Bath, Bath,

$\mathrm{BA} 27 \mathrm{AY}$, England.

Email m.e.loades@bath.ac.uk

(+44) 01225 385249;

BA(Cantab), DClinPsy

This article is a summary of the paper published in $\mathrm{CAMH}$ - Keane, L. \& Loades, M.E. (2017). Low self-esteem and internalizing disorders in young people: A systematic review. Child and Adolescent Mental Health, 22, 4-15. doi: 10.1111/ camh.12204
Young people presenting to CAMHS often report problems with 'low selfesteem'. Self-esteem can be thought of as the overall opinion or evaluation we have of ourselves, including the judgements we make about ourselves and the value we attach to ourselves. Questionnaires like the Rosenberg Self-Esteem Scale can be used to assess self-esteem. There is a complex relationship between self-esteem and mental health problems. It is possible that low self-esteem can be a vulnerability factor, predisposing a person to developing a mental health problem. It is also possible that low self-esteem can result from mental health difficulties and their sequelae (e.g. the negative thinking about the self in depression). This complexity is further complicated by the conceptual overlap between the main constructs of low self-esteem and depression, and their associated measures.

Cognitive Behavior Therapy (CBT) for low self-esteem has shown promise as a broadly applicable intervention in adults, tackling difficulties with anxiety and low mood at the same time. It is based on the CBT model of low selfesteem, developed by Melanie Fennell and colleagues. Treatment is driven by an individualised formulation, and involves identifying and challenging negative beliefs about the self, referred to as the 'bottom line' (e.g. 'I am worthless/unlovable'), and also noticing and logging positive evidence about the self and one's positive qualities and strengths. So far, this approach has not been tested in young people. To make the case to test it, clarity is needed about the extent to which low self-esteem is associated with 'clinically significant' anxiety and depressive disorders in young people (that is, anxiety or depression which meets full diagnostic criteria). Furthermore, it is also important to know the extent to which low self-esteem in young people poses a vulnerability for subsequent mental health difficulties, which would strengthen the argument for treating it as a preventative measure.

Our systematic review aimed to establish what is known about low selfesteem and anxiety/depression in young people $(<18 \mathrm{~s})$. We wanted to find out whether young people with clinically significant anxiety disorders and/or depression also have low self-esteem as measured on validated questionnaires. 
We also wanted to know whether young people with low self-esteem as measured on a validated questionnaire develop depression and anxiety symptomology later in adolescence and young adulthood.

To address these questions, we systematically searched electronic databases to identify relevant studies.

We found 10 studies which looked at the co-occurrence of low self-esteem and clinically significant anxiety/depression. These studies included young people recruited from the community and inpatient and outpatient mental health services. Young people with depression tended to report lower self-esteem than those with anxiety disorders, while those with both anxiety and depression were found to have the lowest self-esteem. Young people with any mental health problems tended to have lower self-esteem than those who do not have mental health problems.

We found 8 studies which reported on the association between low self-esteem in young people and anxiety/depression symptoms in the subsequent 1 to 6 years. From these studies, low self-esteem in young people appeared to be a relatively weak predictor of the development of anxiety and depression in later adolescence and early adulthood. Several studies reported a significant association, although with a relatively small effect, between low self-esteem and subsequent depressive symptoms. For example, Trzesniewski and colleagues (2006) reported that adolescents with low self-esteem were 1.26 times more likely to develop Major Depressive Disorder by the age of 26 than healthy adolescents. This pattern remained even when other vulnerability factors for developing depression (e.g. baseline depression, socioeconomic status and IQ) were taken into account. However, other studies did not find the same association, most likely because the link between self-esteem and later depression is complex and multi-faceted, and likely to be influenced by the accumulation of multiple risk factors. Fewer studies reported on later anxiety. Interestingly, Trzesniewski et al. (2006) found that adolescents with low self-esteem were 1.6 times more likely to develop an anxiety disorder.

The studies we reviewed suggest that young people with clinically significant anxiety or depression, and particularly those with co-morbid anxiety and depression are also likely to have low self-esteem. Further research into the utility of the CBT model of low self-esteem and treatment based on this transdiagnostic model in young people is indicated. A number of smaller scale case series may be helpful to first establish whether a larger trail of CBT is indicated.
Author statement:

Dr Loades is funded by the National Institute for Health Research (Doctoral Research Fellowship, DRF-201609-021). This report is independent research. The views expressed in this publication are those of the authors(s) and not necessarily those of the NHS, The National Institute for Health Research or the Department of Health. 